\title{
A Review on Metabolic Syndrome and Periodontal Disease
}

\author{
Priyalakshmi.S ${ }^{1}$, Sankari.M ${ }^{2}$ \\ Undergraduate student Department of Periodontics, Saveetha Dental College, India \\ ${ }^{2}$ Professor Department of Periodontics, Saveetha Dental College, India
}

\begin{abstract}
Hardly a day goes by in the world of dentistry without some mention of oral systemic relationship. The prevelance of systemic diseases are increasing world wide due to the changes in the life style pattern. It has been concluded that obesity and periodontal disease are associated with each other and obesity has emerged as a risk indicator of periodontal disease. Many studies have been done to find the impact of systemic diseases on oral health and found that there is an association between Diabetes, Obesity and Periodontal disease. Furthermore systemic disorders have been found to have a direct effect on periodontal tissues and these represent the periodontal manifestations of systemic disease. Of late metabolic syndrome and periodontal disease have been linked. Metabolic syndrome is a group of inter-related metabolic abnormalities which increases the risk of cardio vascular morbidity and mortality. So it is important for a dentist to be updated on oral systemic relationship to implement appropriate plans for its prevention and treatment. In this article definition, pathogenesis, about the components of metabolic syndrome and its association with periodontal disease has been reviewed.
\end{abstract}

Key word: systemic inflammation,periodontal pathogens, inflammatory mediators

\section{Introduction}

Metabolic Syndrome is a combination of several metabolic risk factors such as abdominal obesity, high blood pressure, lipid abnormality and hyperglycemia. ${ }^{[1]}$ The metabolic syndrome is also known as Syndrome X, dysmetabolic syndrome, deadly quartet and plurimetabolic syndrome. ${ }^{.2]}$ The changes in lifestyle has increased the prevalence of metabolic syndrome from developed countries to developing countries like India. Metabolic syndrome comprises insulin resistance(fasting blood sugar $>10 \mathrm{mg} / \mathrm{l})$, dyslipidaemia (triacylglycerol $>15 \mathrm{mg} / \mathrm{l}, \mathrm{HDL}<4 \mathrm{mg} / \mathrm{l}$ for $\mathrm{men}$ and $5 \mathrm{mg} / \mathrm{l}$ for women, LDL $>15 \mathrm{mg} / \mathrm{l}$ ), essential hypertension(blood pressure of $>130 \mathrm{mmHg}$ for systolic and sss $>85 \mathrm{~mm} \mathrm{Hg}$ for diastolic)and visceral obesity(waist circumference of $>1020 \mathrm{~mm}$ for men and $>890 \mathrm{~mm}$ for women) ${ }^{[3]}$ According to NCEP ATP III an individual should be considered as having metabolic syndrome when he or she presents with any 3 components of it. ${ }^{[4]}$ It is a well established fact that the primary etiological agent in periodontal disease is bacterial plaque. The toxins and the enzymes produced by the bacterial plaque elicit an inflammatory and immunologic changes in the periodontal tissues at both cellular and molecular levels. Periodontitis is a common chronic infection of the adult population characterized by an exaggerated gingival inflammatory response against a pathogenic bacterial microflora, resulting in alveolar bone loss and eventually tooth loss. ${ }^{[5]}$ Periodontitis is associated with systemic alterations like low-grade inflammation ${ }^{[6]}$ Dyslipidemia ${ }^{[7]}$ glucose intolerance ${ }^{[8]}$ endothelial dysfunction. $^{[9]}$ Lipopolysaccharide derived from periodontal pathogen, Porphyromonas gingivalis which increases the circulating Tumour necrosis factor- $\alpha^{[10]}$ which induces the insulin-resistance and atheromatous changes. ${ }^{[1]}$ In metabolic syndrome there is an elevation in circulating proinflammatory cytokines and a procoagulant state exists with elevation of C-reactive protein and fibrinogen. ${ }^{[12]}$ Both periodontitis and metabolic syndrome are associated with systemic inflammation and insulin resistance with elevation in WBC count as a result of body's defence mechanism. ${ }^{[13]}$ Considering chronic inflammation as the basis for the association between periodontal disease and Metabolic syndrome they can be linked through a common patho physiological pathway. ${ }^{[14]}$ A longitudinal study on 692 BLSA participants performed in the Baltimore, provides evidences that association of alveolar bone loss to Metabolic syndrome is consistent with the hypothesis that destructive periodontal disease may contribute to the development of metabolic syndrome and elevation in systemic inflammation ${ }^{[15]}$

\section{Pathogenesis Of Metabolic Syndrome}

The metabolic syndrome seems to have three potential etiological catogeries like obesity, disorders of adipose tissue, insulin resistance. Other factors like age, gender, ethnicity, diet, physical activity and various unknown genetic factors contribute to the aetiopathogenesis. ${ }^{[16]}$ Proinflammatory cytokines may be a multidirectional link among periodontitis, obesity and other chronic diseases. ${ }^{[17]}$ High dietary intake and low exercise causes imbalance between energy intake and energy expenditure leading to increased waist circumference. The adipose tissues is a large resorvior of biologically active mediators such as Tumour necrosis 
factor $-\alpha$, and other adipokines such as leptin, resistin and adiponectin in inflammatory process. ${ }^{[18]}$ The abundant free fatty acids from expanded adipose tissue decreases insulin sensitivity of muscle cells since adipocytes are unable to take up glucose and store free fatty acids which leads to hyperglycemia. Excess free fatty acids are diverted to liver which alters lipoprotein production in liver thereby causing increase in triglyceride levels and decrease in HDL level. Tumour necrosis factor- $\alpha$ and interleukin- 6 may increase insulin resistance. So pancreas produce additional insulin resulting in hyperinsulinemia. On the other hand leptin causes elevation of blood sugar level by sympathetic nervous system stimulation leading to angiotensinogen release in to blood which causes vasoconstrictive effects on blood vessels, contributing to hypertension. Vascular endothelial growth factor plays a role in atherogenesis and hypertension ${ }^{[19]}$

\section{Pathogenesis Of Periodontal Disease}

Bacterial biofilm is necessary for development of periodontal disease which progresses to loss of gingival insertion and bone resorption as a result of infection by periodontal pathogens such as Porphyiromonas gingivalis, Prevotella intermedia, Tannerella forsythia and Aggregatibacter actiomycetemcomintans ${ }^{[20]}$ which provokes host response leading to release of pro inflammatory mediators resulting in periodontal tissue destruction. $^{[21]}$

\section{Association Between Periodontal Disease And Components Of Metabolic Syndrome. PERIODONTAL DISEASE AND OBESITY[4.1]}

Obesity is defined by the body mass index as greater than $30.0 \mathrm{~kg} / \mathrm{m}^{2}$. Cytokines and hormones from the fat tissue plays an important role in the pathogenesis. ${ }^{[22]}$.Tumor necrosis factor-alpha, leptin, adiponectin and resistin modulates the periodontal response. ${ }^{[23][24]}$ Leptin controls the appetitie, regulates the immune response and the production of inflammatory cytokines. The obesity is associated with the reduction of the sensibility to the effects of the leptin ${ }^{[25]}$ which stimulates the immunological system. ${ }^{[26]}{ }^{[27]}$ In periodontitis there is a negative correlation among the levels of leptin in the gingival crevicular fluid. ${ }^{[27]}$ Adipocytes secreting proinflammatory cytokines may be the molecules linking to the pathogenesis of periodontal infection.

\section{PERIODONTAL DISEASE AND HYPERLIPIDEMIA[4.2]}

Hyperlipidemia has a deregulating effect on the immune-system cells and tissue healing, increasing the susceptibility to infections, such as periodontitis. ${ }^{[28]}$ It has been proved that individuals with periodontal disease have higher serum levels of total cholesterol, low density lipoprotein cholesterol and triglycerides, when compared with periodontally healthy individuals. ${ }^{[29][30]}$ The Alteration in the phenotype of immune cells because of the lipids and the serum elevation of proinflammatory cytokines such as Tumour necrosis factor- $\alpha$ and Interleukin-16 from chronic periodontitis evidenced the bidirectional relationship between the two conditions. ${ }^{[31]}$

\section{PERIODONTAL DISEASE AND HYPERTENSION[4.3]}

The periodontal disease may stimulate the systemic inflammation linked to Cardiovascular disease. Moreover, the chronic inflammation and the inflammatory cytokines may cause endothelial dysfunction establishing a connection between inflammation and risk for cardiovascular disease. This connection could be mediated by alterations in the vascular resistance causing blood pressure. ${ }^{[32]}$

\section{PERIODONTAL DISEASE AND INSULIN RESISTANCE /DIABETES TYPE 2[4.4]}

Diabetes and periodontal disease are two chronic diseases that have been considered as biologically connected ${ }^{[33]}$ Hyperglycemia and the formation of the advanced glycation end-products (AGEs) are some of the several possible ways leading to the classical vascular complications of diabetes, also involved in the physiopathology of periodontitis in diabetic individuals. ${ }^{[34]}$ Diabetic patients are more susceptible to develop periodontal disease because of the polymorphonuclear leukocytes disease, alterations in the collagen metabolism. The formation of AGEs affects the collagen stability and the vascular integrity. AGEs aggregate macrophage and monocyte receptors of interleukin-l and Tumour necrosis factor- $\alpha$, which provokes an increase of susceptibility to periodontal disease. ${ }^{[35]}$ Inflammatory cytokines induce the insulin resistance and the chronic inflammatory diseases, including periodontitis ${ }^{[36]}$ Additionally both Tumour necrosis factor- $\alpha$ and Interleukin- 6 is derived from the fat tissue which mediates inflammation, suggesting that obesity, diabetes and periodontitis are mutually related.

\section{IMPACT OF PERIODONTAL INFECTION ON SYSTEMIC HEALTH.[4.5]}

Periodontal disease and metabolic syndrome has bidirectional association.Not only systemic diseases have impact on oral health but oral diseases like periodontal diseases have wide ranging systemic effects. In susceptible individuals, periodontal infection acts as an independent risk factor for systemic diseases and may be 
involved in the basic mechanism of these conditions. The pathogenic bacteria alone is not sufficient to create an disease rather the disease initiation and progression also depends on host susceptibility.Periodontitis is an chronic inflammatory disease caused by periodontal pathogens in presenceof local factors which is significantly associated with the frequency of tooth brushing,regular dental visits, smoking and drinking habits ${ }^{\left[{ }^{[3]}\right.}$ The consequence of chronic periodontal infection is the synthesis of inflammatory cytokines and chemokines by local periodontal tissue or circulating inflammatory cells. The inflammatory cytokines such as interleukin-6, Tumour necrosis factor- $\alpha$, elevated C-reactive protein levels ${ }^{[38]}[39]$. evokes systemic inflammation which contributes to insulin resistance and causes hyperglycemia,which in turn induces the expression of proinflammatory mediators and increases the risk of oral infection and cardiovascular disease among patients with periodontitis.Thus a triangular vicious circle among periodontitis,systemic inflammation and hyperglycemia is perpetuated. ${ }^{[40]}$ The prevelance of periodontitis is doubled in patients with diabetes and thus it is commonly interpreted that diabetes affects the development of periodontitis.However the reverse possibility that periodontitis disturbs glucose handling is also reasonable and worthy of consideration. The periodontitis can also be associated with dyslipidemia via systemic inflammation.Many cytokines including Interleukin6,Interleukin-1,Tumour necrosis factor- $\alpha$,stimulate hepatic free fatty acid synthesis resulting in increased synthesis of low density protein and hypertriglyceridemia ${ }^{[41]}$ Moreover excessive intracellular fatty acids can increase the production of reactive oxygen species, which activate the proinflammatory nuclear factor kappa B pathway and thereby increase the inflammatory response and insulin resistance. Thus periodontitis may also start vicious cycle involving systemic inflammation and increased synthesis of free fatty acids ${ }^{.[42][43]}$

\section{Significance Of Maintaining Oral Health}

Oral cavity is said to be the mirror of the systemic health.The manifestation of systemic diseases are seen in oral cavity.On the other hand oral diseases has an potential to act as an independent risk factor for causing systemic diseases.On linking metabolic syndrome and periodontal disease, maintainence of good oral health can reduce the prevelance of metabolic syndrome.The good oral health can be achieved by proper brushing.The main purpose of tooth brushing is ,the disturbance of the biofilm and bacterial colonies as well as removal of dental plaque to prevent caries and periodontal disease since periodontal disease is associated with frequency of tooth brushing[44] ][45].Low tooth brushing frequency is associated with elevated c-reactive protein levels and fibrinogen levels suggesting systemic inflammatory status ,enhancing the risk of cardiovascular disease and metabolic syndrome. [46]

\section{Management}

The International Diabetes Federation recommended that the management of the metabolic syndrome must be aggressive and uncompromising in its aim to reduce the risk of cardiovascular disease and type 2 diabetes. The prime emphasis in individuals with metabolic syndrome is to mitigate the underlying risk factors through life style changes ${ }^{[47]}$.ATP recommended that obesity be the primary target of intervention for metabolic syndrome.First line of therapy should be weight reduction with increased physical activity.Weight loss lowers serum cholesterol and triglycerides,raises HDL cholesterol,lowers blood pressure and glucose and reduces insulin resistance.In people for whom life style changes is not enough, drug therapy may be required. It is necessary to treat individual components of metabolic syndrome in order to reduce the individual risk associated with each component. ${ }^{\left[{ }^{[8]}\right]}$.However the meticulous treatment of metabolic syndrome should not only be a prime concern for terminating disease pathogenesis but also periodontitis should be treated. The presence of deep periodontal pockets implies the existence of current local inflammation in periodontal tissue.The removal of the plaque and the factors that favours its accumulation is the primary goal in periodontal therapy.The periodontal therapy comprising of oral prophylaxis, gingival curettage, flap surgery ,mucogingival surgery has to be performed when required to restore periodontal health which in turn improves systemic health since periodontal treatment reduces Tumour necrosis factor- $\alpha$ and $\mathrm{C}$-reactive protein levels thereby minimizing the effect of the periodontal health on metabolic syndrome.Education and motivation to the patient ,followed by recalls also favours a path to good prognosis. Controlling the periodontal disease has positive results on reducing metabolic syndrome. So preservation of the periodontal health of the treated patient have to be maintained by Supportive periodontal treatment ${ }^{[48]}$

\section{Conclusion}

Hence this review article provides an wide description on association between oral and systemic diseases. The observed association is compatible with the hypothesis that chronic inflammation is an important factor in the physiopathology underlying periodontal disease and metabolic syndrome. The awareness on association of systemic oral relationship would throw an insight on life style modifications. Further investigations are required to determine whether oral health care in individuals exhibiting metabolic syndrome has the potential to reduce the incidence of various systemic diseases. 


\section{References}

[1] Nao Fukui, Yoshihiro Shimazaki, Takashi Shinagawa and Yoshihisa Yamashita,Periodontal Status and Metabolic Syndrome in Middle-Aged Japanese,J Periodontol,83, 2012,1363-1371.

[2] Alexandrina L.Dumitrescu, L.Zetu, Silvia Teslaru, Metabolic syndrome and Periodontal diseases.ROM.J.Intern.Med,46(3) ,2008,207-212.

[3] Alexandrina L.Dumitrescu, L.Zetu, Silvia Teslaru, Metabolic syndrome and Periodontal diseases.ROM.J.Intern.Med, 46(3) 2008,207-212.

[4] National cholesterol Education Program Expert panel on detection, evaluation, and treatment of high blood cholesterol in adults (Adults treatment panel II I) JAMA,16.285(19),2001 May,2486-97.

[5] Williams RC 1990 Periodontal disease. N Eng J Med 322:373-382.

[6] Slade GD, Offenbacher S, Beck JD, Heiss G, Pankow JS 2000 Acute -Phase inflammatory response to;periodontal disease in the US population. J Dent Res 79;49-57.

[7] Katz J Flugelman MY, Goldberg A, Heft M2002 Association between periodontal pockets and elevated cholesterol and low density lipoprotein cholesterol levels. J periodontal 73,494-500.

[8] Saito T, Shimazaki Y, Kiyobara Y, kato I, Kubo M, Lida M, koga T, The severity of periodontal disease is associated with the development of glucose intolerance in non-diabetics ,the Hiasayama study, J dent Res 83,2004,485-490.

[9] Tonetti MS D Aiuto F, Nibali L, Donald A, Storry C, Parkar M, Suvan J, Hingorani AD Vallance P, Deanfield ,Treatment of periodontitis and endothelial function N Engl J Med 356 ,2007,911-920.

[10] Lang $\mathrm{CH}$ Dobrescu C, Bagby Gj,Tumor necrosis factor impairs insulin action on peripheral glucose disposal and hepatic glucose output. Endocrinology 1992;130:43-52.

[11] Page RC. The pathobiology of periodontal diseases may affect systemic disease. Invertion of a paradigm. Ann periodontal 1998;3:108-120.

[12] Ford ES 2003 The metabolic syndrome and C-reactive protein, Fibrinogen and leukocyte count:findings from the Third national Health and Nutrition Examination Survey:Atherosclerosis 168:351-358.

[13] Nakanishi N, Suzuki K, Tatara K. White blood Cell count and clustered features of metabolic syndrome in Japanese male officers. Occup Med (Lond);52,2002,213-218.(pubMed:12091587).

[14] Mark J. Nesbitt Mark ${ }^{1}$ A. Reynolds ${ }^{2}$, Harian Shiau ${ }^{2}$, Kyong Choe ${ }^{2}$, Eleanor M. Simonsick ${ }^{2}$ and Luigi Ferrucci ${ }^{1}$, Association of periodontitis and metabolic syndrome in the Baltimore Longitudinal Study of Aging Aging Clin Exp Res.;22(3),2010 June ,238242.

[15] Mark J. Nesbitt Mark ${ }^{1}$ A. Reynolds ${ }^{2}$, Harian Shiau ${ }^{2}$, Kyong Choe ${ }^{2}$, Eleanor M. Simonsick ${ }^{2}$ and Luigi Ferrucci ${ }^{1}$ Association of periodontitis and metabolic syndrome in the Baltimore Longitudinal Study of Aging. Aging Clin Exp Res,22(3) ,2010 June,238242 .

[16] Vipin Bharti, Pankaj Khurana. Metabolic syndrome and periodontal disease. Jounal of Indian society of Periodontology,13(3),2009Sep,172-174.

[17] SAITO T, SHIMAZAKI Y. Metabolic disorders related to obesity and periodontal disease. Periodontol, ,43, 2000,2007,254.

[18] SAITO T, SHIMAZAKI Y. Metabolic disorders related to obesity and periodontal disease. Periodontol, ,43, 2000,2007,254.

[19] Vipin Bharti, Pankaj Khurana. Metabolic syndrome and periodontal disease. Jounal of Indian society of Periodontology,13(3),2009Sep,172-174.

[20] Genco RJ. Current view of risk factors for periodontal diseases J periodontal;67(10suppl) 1996,1041-9

[21] Andriankaja OM, Sreenivasa S, Dunford R, DeNarin E Association between metabolic syndrome and periodontal disease. Austr Dent J. 2010 Sep5(3):252-259.

[22] Pischon N Heng N, Berimoulin JP, Kleber BM, Willich SN, Pischon T. Obesity, inflammation and periodontal disease J Dent Res,86(5)May2007,400-4009.

[23] Martin SS, Qasim A, Reilly M.J. Leptin resistance: A possible interface of inflammation and metabolism in obesity-related cardiovascular disease. Am coll Cardiol,;52(15), 2008Oct ,1201-10.

[24] Saito T,Shimazaki Y.Metabolic disorders related to obesity and periodontal didease.Periodontol;43, 2000.2007,254-266.

[25] Pischon N, Heng N, Bernimoulin JP, Kieber BM, Willich SN, PIschon T, Obesity, inflammation and periodontal disease J Dent Res, 86(5),2007May,400-4009.

[26] Saito T, Shimazaki Y Metabolic disorders related to obesity and periodontal disease Periodontol 43, 2000,2007,254-66.

[27] Karthikyan BV, Pradeep AR, Gingival Crevicular fluid and serum leptin:their relationship to periodontal health and disease J Clin periodontal 34(6), 2007 Jun,467-472.

[28] Fentoglu O, Bozkurt FY, The bidirectional relationship between periodontal disease and hyperlipidemia Eur J Dent 2(2), 2008 Apr,142-6.

[29] Fentoglu O, Koroglu BK Hicyilmaz H, Sert T, Oxdem M. Sutcu R et al Pro inflammatory cytokine levels in association between periodontal disease and hyperlipidemia J Clin Periodontol 38(1), 2011 Jan,8-16

[30] Hamissi J Shahsavarani MT, Shahsavarani H, Sayahpour S, Hamissi H. A comparison of the serum lipid level between patients with periodontitis and healthy individuals. J periodontal Implant Dent 2(1) 2010,29-32.

[31] Fentoglu O, Bozkurt FY. The bi-directional relationship between periodontal disease dand hyperlipidemia EUr J Dent;2(2), 2008 Apr,142-146

[32] Tsioufis C, Kasiakogias A, Thomopoulos C, Stefanadis C. Periodontitis and blood pressure :the concept of dental hypertension. Atherosclerosis 2011 Nov;219(1):1-9.

[33] Nagasawa T, Noda M Katagiri S, Takaichi M, Takahshi Y, Wara-Aswapati N et al. Relationship between periodontitis and diabetes - importance of a clinical study to prove the vicious cycle. Inter Med. 2010;49(10):881-5.

[34] Mealey BL, Rose LF. Diabetes mellitus and inflammatory periodontal diseases. Curr Opin Endocrinal Diabetes Obes.2008 Apr;15(2):135-41

[35] Lazenby MG, Crook MA The innate immune system and diabete mellitus:the relevance of periodontitis? A hypothesis. Clin Sci. 2010 Aug:119(10)423-9.

[36] Mealey BL. Ocampo GI. Diabetes mellitus and periodontal disease Periodontol 2000.2000;44:127-53.

[37] Y. Shimazaki,T.Saito,K.Yonemoto,Y.kiyohara,M.Lida and Y.Yamashita.Relationship of Metabolic syndrome to Periodontal Disease inJapanese Women:The Hisayama study.JDent Res 86(3):271-275,2007.

[38] Chen LP,Chaing CK,Chan CP et al.Does periodontitis reflect inflammation and malnutrition status in hemodialysis patients?Am J Kidney Dis;47; 2006,s815-822.

[39] Fentoglu O,Koroglu BK,Hicyilmaz $\mathrm{H}$ et al.Pro inflammatory cytokine levels ion association between periodontal disease and hyperlipidaemia.J Clin Periodontol 38,8-16 
[40] Kirk EP,Klien S.Pathogenesis and pathophysiology of the cardiometabolic syndrome.J Clin Hpertens(Greenwich )11,2009,761765 .

[41] Khovidhunkit W,Memon RA,Feingold KR et al.Infection and inflammation-induced proatherogenic changes of lipoproteins.J Infect Dis 2000;181(Suppl 3):S462-S472.

[42] Itani SI,Ruderman NB,Schmiieder F et al.Lipid induced insulin resistance in human muscle is associated with changes in diacylglycerol,protein kinase C,and IkappaB-alpha.Diabetes 2002;51:2005-2011

[43] Steinberg Ho,Tarshoby M,Monestel R et al.Elevated circulating free fatty acid levels impair endothelium-dependent vasodilation.J Clin Invest 1997;100:1230-1239

[44] Lang WP,Ronis DL,Farghaly MM.Preventive behaviours as correlates of periodontal health status.J Public Health Dent 1995;55:1017

[45] Vysniauskaite S,Vehkalahti MM.Impacts of tooth-brushing frequency on periodontal findings in a group of elderly Lithuanians.Oral Health Prev Dent 2009,129-136.

[46] de Oliveira C,Watt R,HAMER m.Tooth brushing,inflammation,and risk of cardiovascular disease;Results from Scottish Health Survey.BMJ2010;340:c2451.

[47] Vipin Bharti, Pankaj Khurana. Metabolic syndrome and periodontal disease. Jounal of Indian society of Periodontology. Sep;13(3), $2009172-174$

[48] Scott M.Grundy ,MD,Phd ;H.Bryan Brewer,Jr.,MD;James I.Cleemann,MD;Sidney C.Smith,Jr,MD;Claude Lenfant, MD;Definition of metabolic syndrome.doi:10.1161/01.CIR.0000111245.75752.C6a 